\title{
PLANTAS MEDICINALES Y PARA CONDIMENTO USADAS EN EL SUDESTE DEL PARTIDO DE BERISSO (BUENOS AIRES, ARGENTINA)
}

\author{
MARCELO P. HERNÁNDEZ ${ }^{1,2,3}$, MARÍA C. NOVOAㄴ, ANA M. ARAMBARRI ${ }^{1}$ \& MARÍA A. OVIEDO³
}

\begin{abstract}
Summary: Hernández, M. P., M. C. Novoa, A. M. Arambarri \& M. A. Oviedo. 2015. Medicinal and condiment plants used in the Southeastern Berisso (Buenos Aires, Argentina). Bonplandia 24(2): 125-138.

In this paper the objective was to survey therapeutic plants used by the inhabitants in a coastal region located along Rio de la Plata and between the riverside resort of Bagliardi and La Balandra. Thirty six species used in folk medicine were recorded among them ten are also used as condiment.
\end{abstract}

Key words: Argentina, condiment, economic botany, popular medicine, Rio de la Plata shore, vascular plants.

\begin{abstract}
Resumen: Hernández, M. P., M. C. Novoa, A. M. Arambarri \& M. A. Oviedo. 2015. Plantas medicinales y para condimento usadas en el sudeste del Partido de Berisso (Buenos Aires, Argentina). Bonplandia 24(2): 125-138.

El objetivo de este trabajo fue relevar las plantas usadas con fines terapéuticos por los habitantes de un sector costanero del Río de la Plata entre los balnearios Bagliardi y La Balandra. Se identificaron 36 especies utilizadas en la medicina popular, 10 de las cuales también se usan como condimento.
\end{abstract}

Palabras clave: Argentina, botánica económica, condimentos, costa del Río de la Plata, medicina popular, plantas vasculares.

\section{Introducción}

El humedal rioplatense situado al sudeste del Partido de Berisso (provincia de Buenos Aires, Argentina), presenta diversos tipos de vegetación espontánea: céspedes ribereños, matorrales y pajonales costaneros y bosques hidrófilos nativos relictos que, en algunos sectores, incluyen especies pertenecientes a antiguas selvas marginales. Afines del siglo XIX y principios del siglo $\mathrm{XX}$, estas selvas, junto a los bosques nativos, se extendían desde NE al SE de la provincia de Buenos Aires, desde la localidad de Punta Lara (Partido de Ensenada) hasta la localidad de Magdalena (Partido de Magdalena) (Cabrera, 1949; Cabrera \& Dawson, 1944; Arturi et al., 2006; Delucchi \& Torres Robles, 2006). La zona presenta una gran riqueza florística, un clima templadocálido $\mathrm{y}$ húmedo con inviernos benignos $\mathrm{y}$ precipitaciones con una media anual de 800$1.000 \mathrm{~mm}$ (Lahitte et al., 1997, 1998). El área

${ }^{1}$ Morfología Vegetal, Laboratorio de Morfología Comparada de Espermatófitas (LAMCE).

${ }^{2}$ Sistemática Vegetal, Facultad de Ciencias Agrarias y Forestales, C.C.31, 1900 La Plata.

${ }^{3}$ Botánica Sistemática II, Facultad de Ciencias Naturales y Museo de La Plata, Universidad Nacional de La Plata, Argentina. Autores para correspondencia: E-mail: mphciencia@yahoo.com o anaramba@yahoo.com.ar 
ha sufrido modificaciones realizadas por el hombre (introducción de especies exóticas, explotación de conchilla, actividad frutícola, hortícola y ganadera, actividad industrial y urbanismo). La población es de ascendencia europea y desarrolla su actividad dentro del área de estudio, tanto en cultivos forestales (sauces y álamos en la región próxima de Palo Blanco y Los Talas), en el cultivo de frutales y plantas hortícolas, así como en la pesca.

Existen estudios previos sobre el uso que los habitantes de otros sectores de la ribera platense hacen de las plantas nativas y exóticas (Buet et al., 2010, 2011; Hernández et al., 2009, 2010, 2013; Hernández \& Arambarri, 2011; Hurrell et al., 2011). Estos trabajos, al igual que el presente, se enmarcan dentro de la botánica económica que Pochettino (2015) define como el "estudio de los vegetales al servicio del hombre", refiriéndonos al beneficio logrado por el hombre tanto de la vegetación natural como cultivada para curar sus dolencias. Así, el objetivo en el presente estudio fue relevar las plantas utilizadas como medicinales por los habitantes de un sector costanero del Río de la Plata localizado entre los balnearios Bagliardi y La Balandra.

\section{Materiales y Métodos}

En el sector costanero del Río de la Plata localizado entre los balnearios Bagliardi y La Balandra (Fig. 1), entre los años 2012-2014 se realizaron encuestas durante cuatro viajes de campaña, uno por cada estación del año, considerando la fenología de las plantas. El segundo año sirvió para repetir las encuestas $\mathrm{y}$, ampliar y confirmar la información lograda durante el primer año. Se aplicaron en el área de estudio distintas técnicas de relevamiento de datos. Se tomó como unidad de análisis la Unidad Doméstica(UD), la cual se puede definir operativamente como la unidad elemental para la descripción de la vida grupal. Esta unidad a veces estuvo constituida por una persona adulta y otras por varias personas, en este último caso se encuestó al hombre o mujer adultos a quienes se les dio el nombre de Jefe o Jefa de familia de esta unidad compleja que incluye el grupo de personas que comparten la residencia, el espacio físico que habitan, articulados por un conjunto de actividades relevantes a la subsistencia del grupo. Se estudiaron 40 UD de informantes legos de ambos sexos que viven en casas distribuidas al azar dentro del área de estudio o sobre los caminos hacia el Río de la Plata. El material botánico y la información obtenidos se relevaron mediante el empleo de la metodología etnográfica, con la aplicación de las técnicas de: (1) selección de la muestra mediante sondeos empíricos en las que se incluyeron informantes legos, (2) observación participante con entrevistas abiertas e informal-estructuradas, (3) recolección de vegetales útiles y (4) registro de la información. Las entrevistas utilizadas se refirieron a las enfermedades reconocidas por los entrevistados y las plantas usadas para su tratamiento, los nombres con que designan estos vegetales, como los obtienen y las partes de las plantas que utilizan, formas de preparación y administración. La información fue registrada mediante un diario de campo, grabaciones de audio y fotografías (Pochettino et al., 1997, 2008; De Paula et al., 2002). Con los jefes/as de la UD se realizó la recolección del material. La información obtenida fue procesada en el laboratorio, donde el material fue identificado por su morfología externa haciendo uso de un microscopio estereoscópico XTX - 7C W y claves dicotómicas. Se emplearon para las especies indígenas Cabrera \& Zardini (1978) y para las especies cultivadas Dimitri (1978, 1980). Los ejemplares recolectados y herborizados fueron incorporados a las colecciones de los herbarios LPAG (Facultad de Agronomía, UNLP) y LP (Facultad de Ciencias Naturales y Museo, UNLP). En algunos casos, una vez identificado el material se prefirió no recolectarlo por su escasez en la UD, en tal situación se utilizó como material de referencia ejemplares de herbario de especies que fueron recolectadas en trabajos previos en las localidades vecinas de Palo Blanco, Los Talas e Islas Santiago y Paulino o excepcionalmente ejemplares existentes en los herbarios LP y LPAG. Para la nomenclatura botánica se consideraron las bases de datos del Instituto de Botánica Darwinion (2015) y Tropicos.org (2015). Los resultados se incluyen en Tablas y Figuras. 


\section{Resultados}

En la Tabla 1 se indican las 36 especies de plantas vasculares usadas en medicina popular, las que fueron ordenadas por familia, se indica el nombre científico, nombre vernáculo según los entrevistados, origen de la especie, hábito, parte del vegetal utilizada, forma de preparación y de administración. En las dos últimas columnas se compara el uso medicinal popular registrado y la actividad biológica evaluada de acuerdo con la bibliografía consultada a ese respecto.

De las 36 especies medicinales relevadas resultó que diez también son utilizadas como condimento (Aloysia citrodora, A. polystachya, Laurus nobilis, Mentha x piperita, Ocimum basilicum, Origanum vulgare, Petroselinum crispum, Rosmarinus officinalis, Salvia officinalis y Thymus vulgaris).

De las especies relevadas predominan las herbáceas (Fig. 2) y se utilizan con mayor frecuencia las especies exóticas cultivadas respecto a las nativas (Fig. 3). Se reconocieron 20 propiedades o usos medicinales locales en las especies estudiadas (Fig. 4), entre los cuales predomina el tratamiento de trastornos digestivos (Tabla 2).

Los habitantes locales obtienen el material vegetal de plantas medicinales en forma manual, directamente de las especies espontáneas que crecen en la zona o de las cultivadas en sus jardines.

De las partes vegetales utilizadas (hojas, partes aéreas, planta entera, flores, frutos, tallos y tubérculos), las hojas son las más utilizadas (Tablas 1 y 3 ).

Se registraron los siguientes modos de preparación: infusión, decocción, decocción acuosa con azúcar, jarabe con azúcar negra y miel, hoja picada, macerado acuoso, pulpa, rodajas de tubérculo y tintura. La infusión es el modo de preparación más frecuente (Tablas 1 y 4$)$.

Se registraron los siguientes modos de administración: oral-caliente, tópico-frío, tópico-tibio, inhalación-vapor, oral-frío, oral-masticación y oral-tibio. El modo de administración más frecuente es oral-caliente (Tablas 1 y 5). Es también importante el uso como tópico-frío (Tabla 5).

Los extractos vegetales del $80 \%$ de las especies reportadas presentan actividad farmacológica relacionada al uso medicinal popular en el área de estudio (Tabla 1).

\section{Discusión}

Se encontró que los trastornos digestivos, epidérmicos y respiratorios son las dolencias

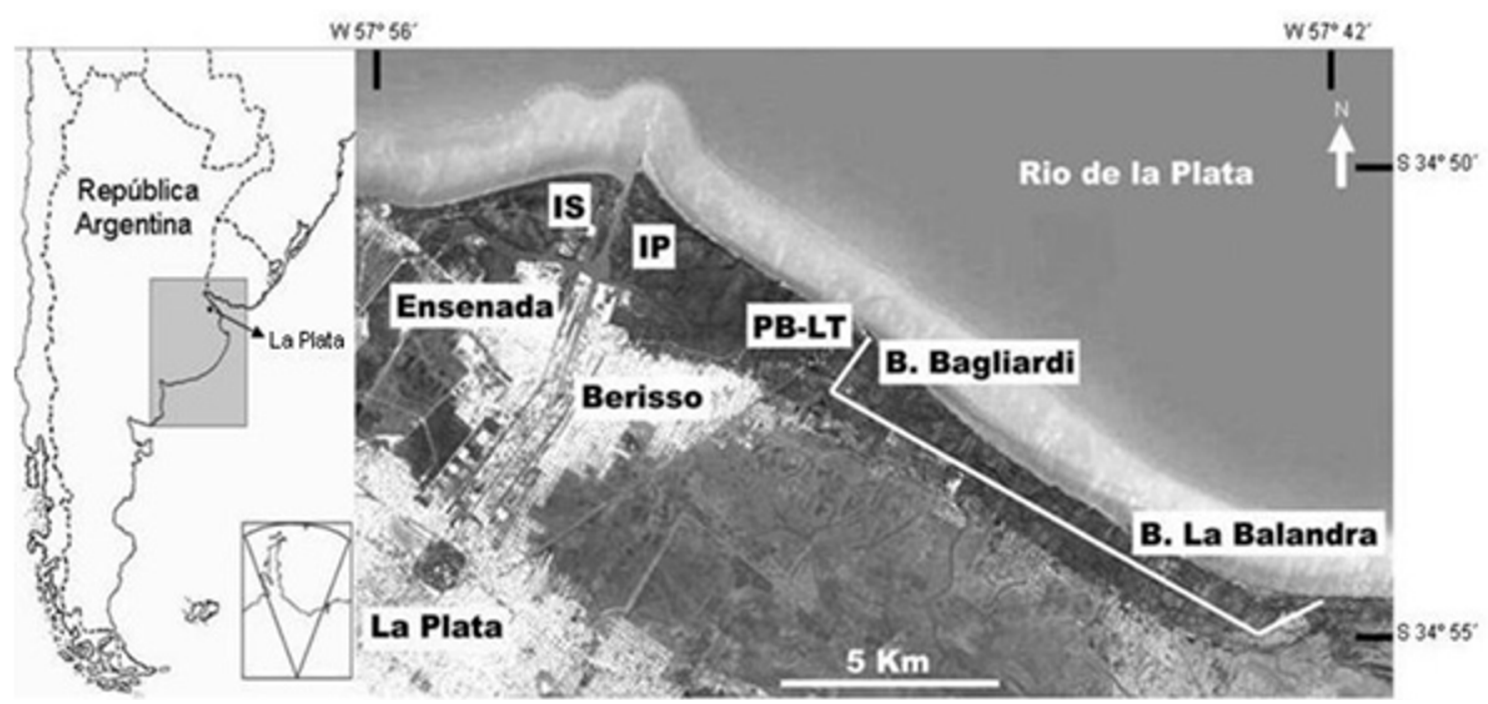

Fig. 1. Área de estudio: región costera del Río de la Plata entre los balnearios Bagliardi y La Balandra. Ref.: B. = balneario; IP = isla Paulino; IS= isla Santiago; PB- LT = Palo Blanco-Los Talas. 


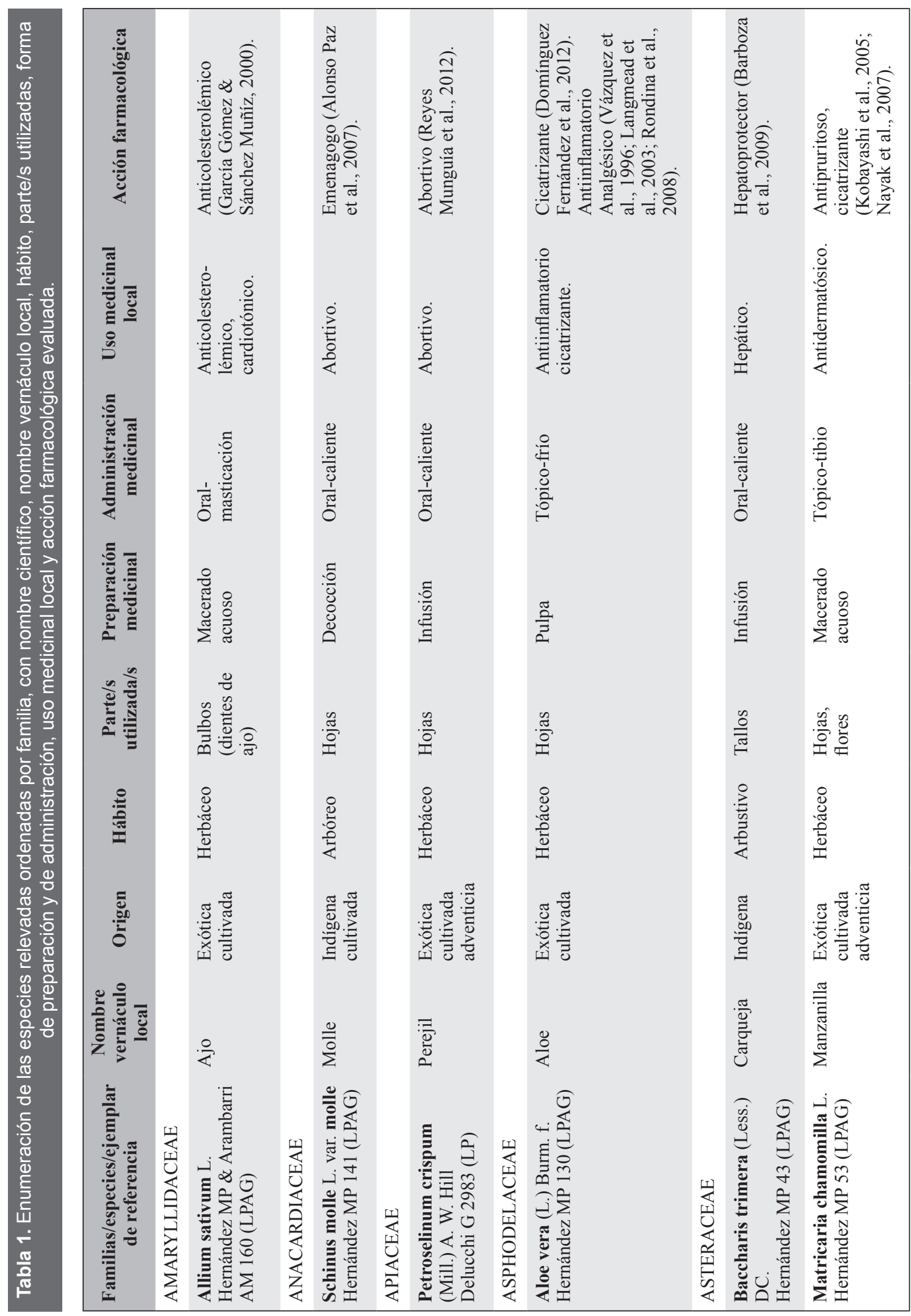




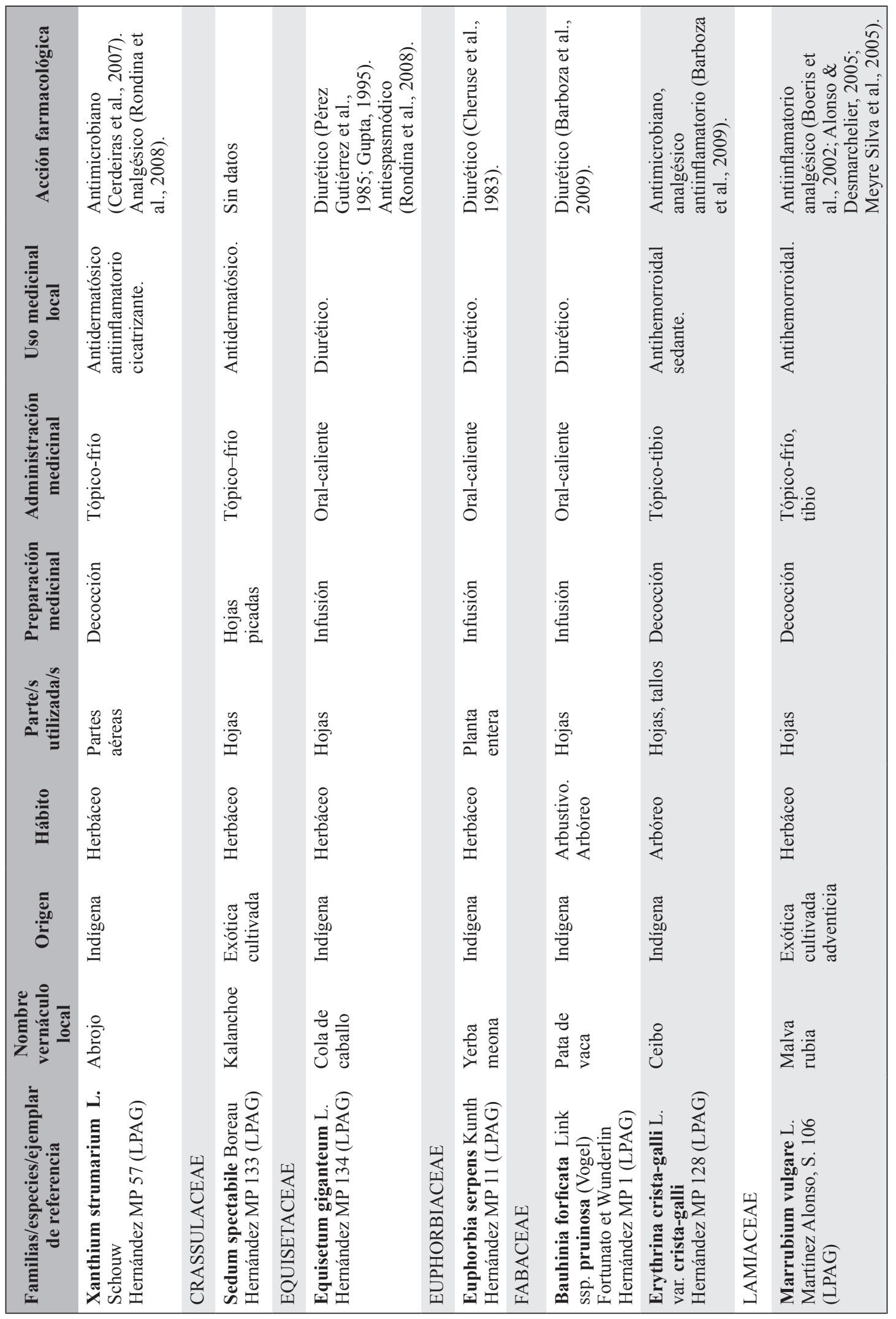




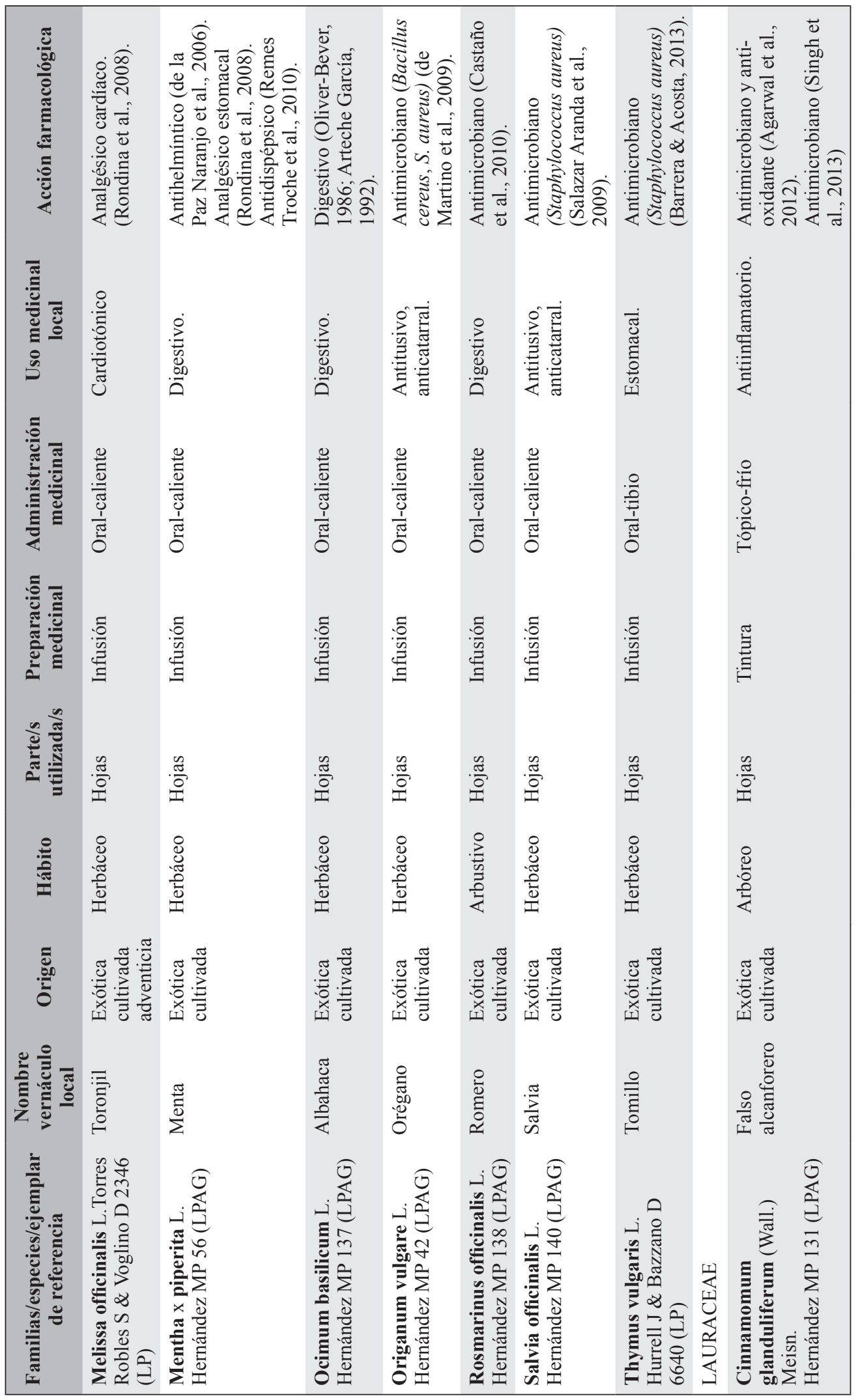




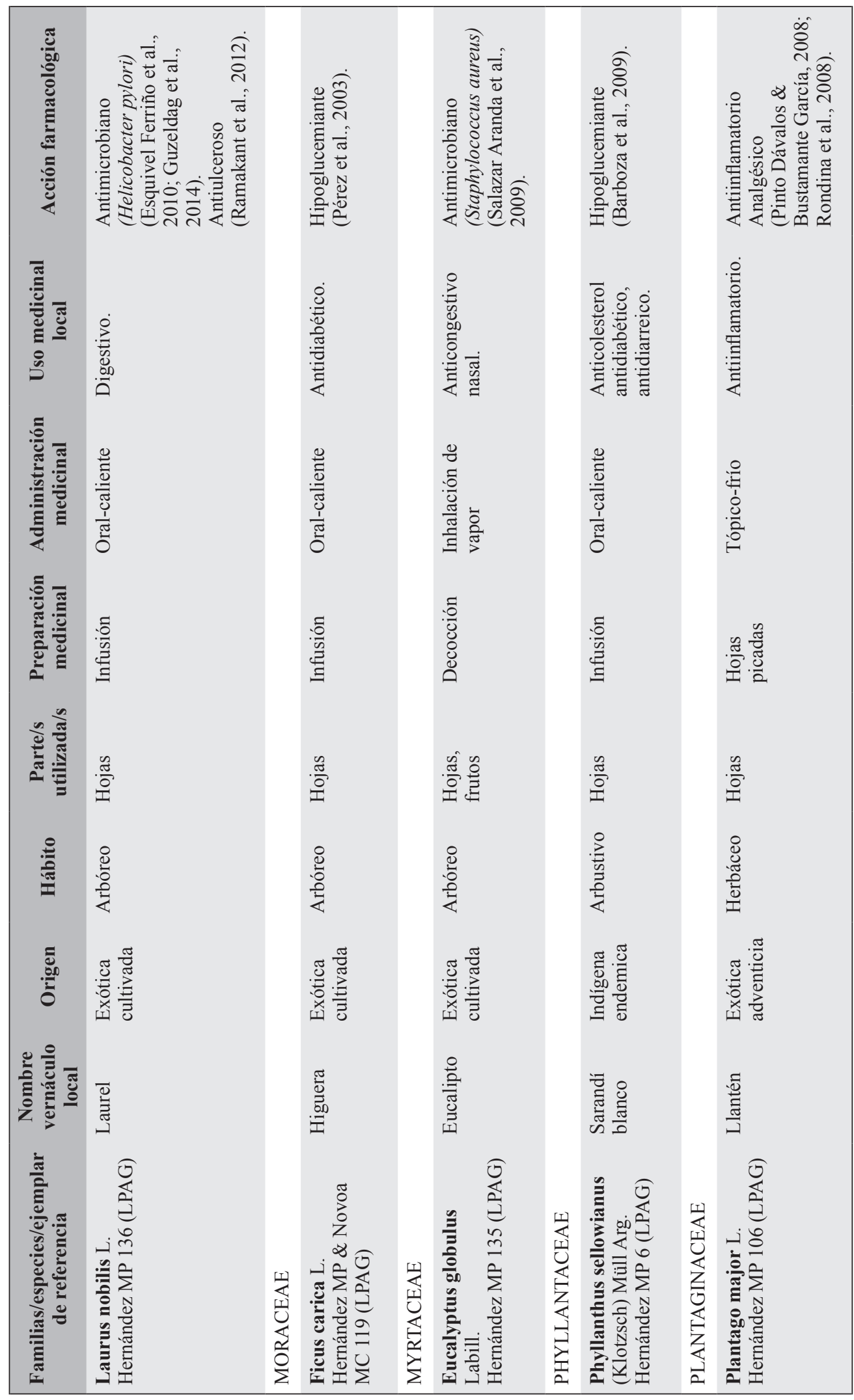




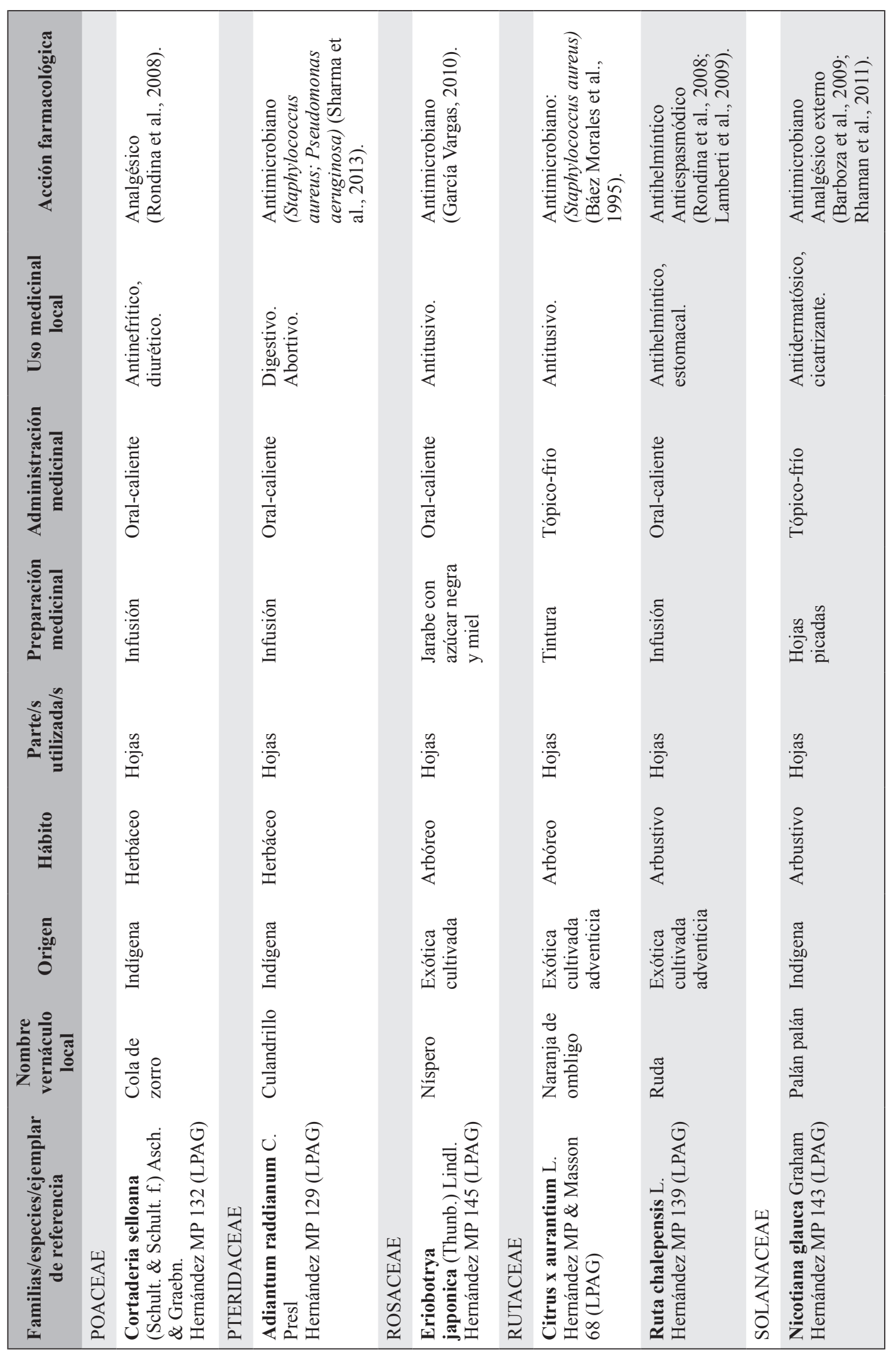




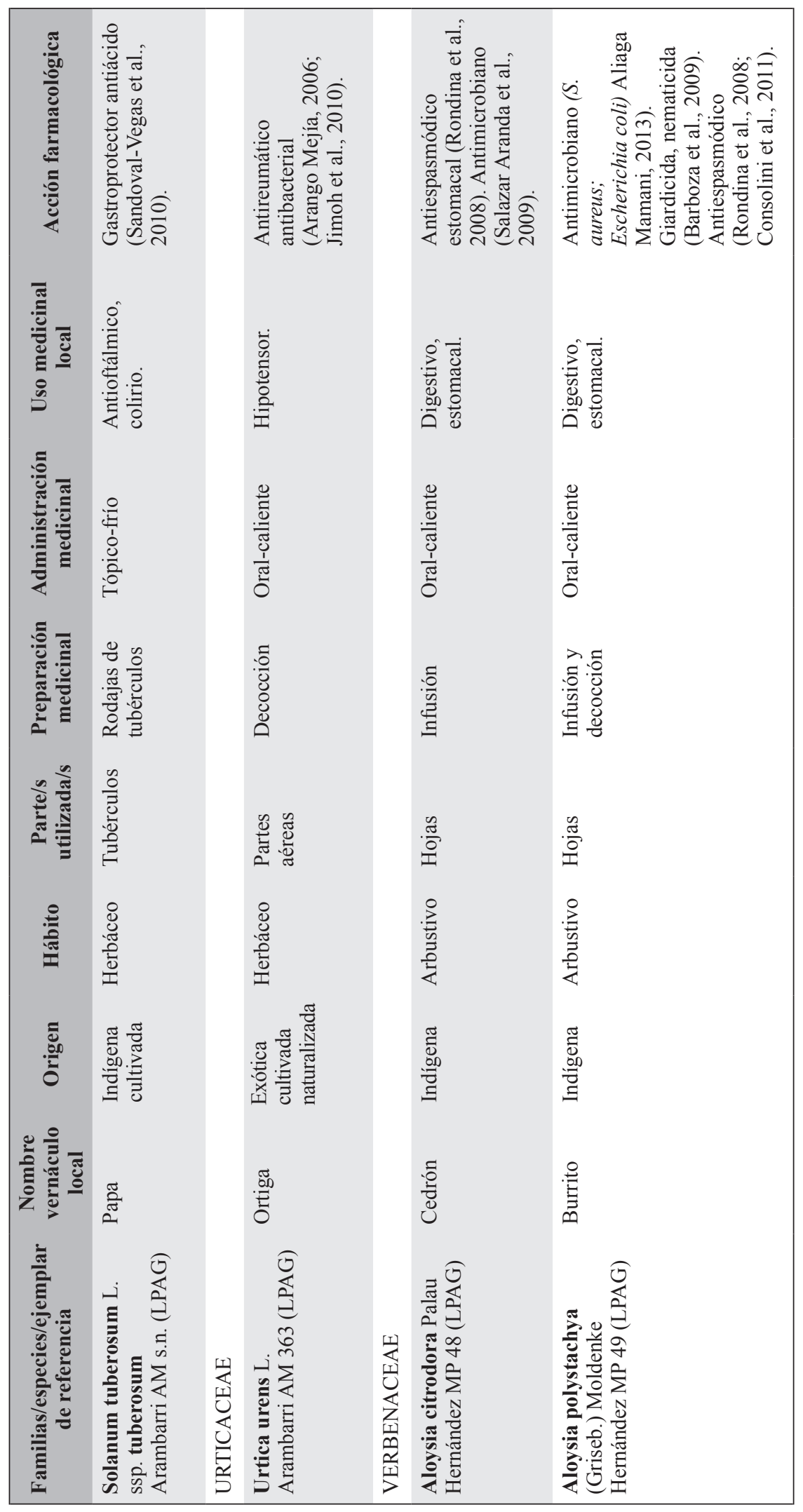




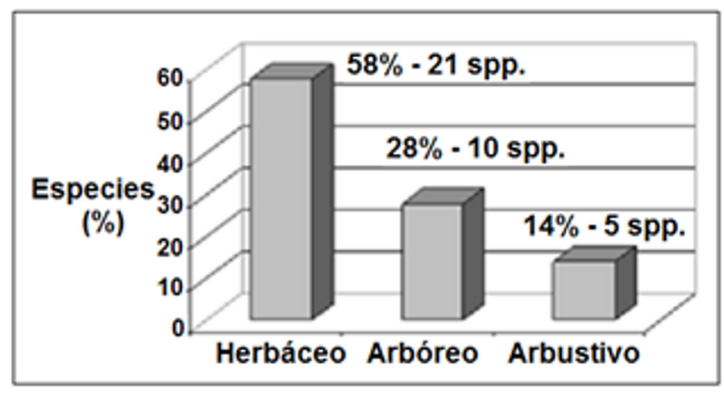

Fig. 2. Hábito de las especies vegetales con uso medicinal local.

tratadas con mayor frecuencia, como se halló previamente para otras localidades próximas de la misma ribera platense (Hernández et al., 2009, 2010, 2013). La población posee conocimiento, en especial sobre las plantas exóticas cultivadas, lo cual se debe a la cultura traída por los inmigrantes europeos en el siglo $\mathrm{XIX}$, quienes ingresaron conjuntamente con sus especies hortícolas, frutales, ornamentales y/o medicinales, como lo expresara Cabrera (1949). Asimismo, la presencia de plantas cultivadas se atribuye también a la proximidad del área con centros urbanos donde pueden ser adquiridas, teniendo muchas de estas una gran

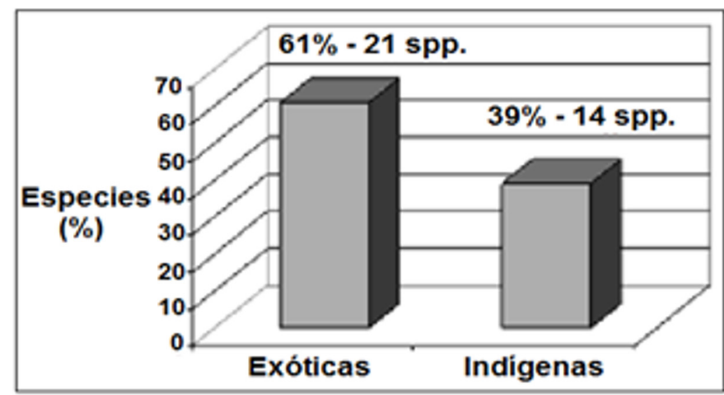

Fig. 3. Origen geográfico de las especies medicinales locales.

capacidad de aclimatación, fácil reproducción y/o multiplicación. Todo esto se corresponde con los datos etnobotánicos registrados para las isla Santiago (Ensenada), isla Paulino y las localidades de Palo Blanco y Los Talas (Berisso) (Buet et al., 2010, 2011; Hernández et al., 2009, 2010, 2013; Hurrell et al., 2011). Entre las especies estudiadas cabe destacar que Adiantum raddianum, Allium sativum, Sedum spectabile y Solanum tuberosum, si bien fueron reportadas como medicinales en otra áreas de estudio (e.g. Pochettino et al., 1997) es la primera vez que se registran considerando las áreas vecinas relevadas de las islas Santiago

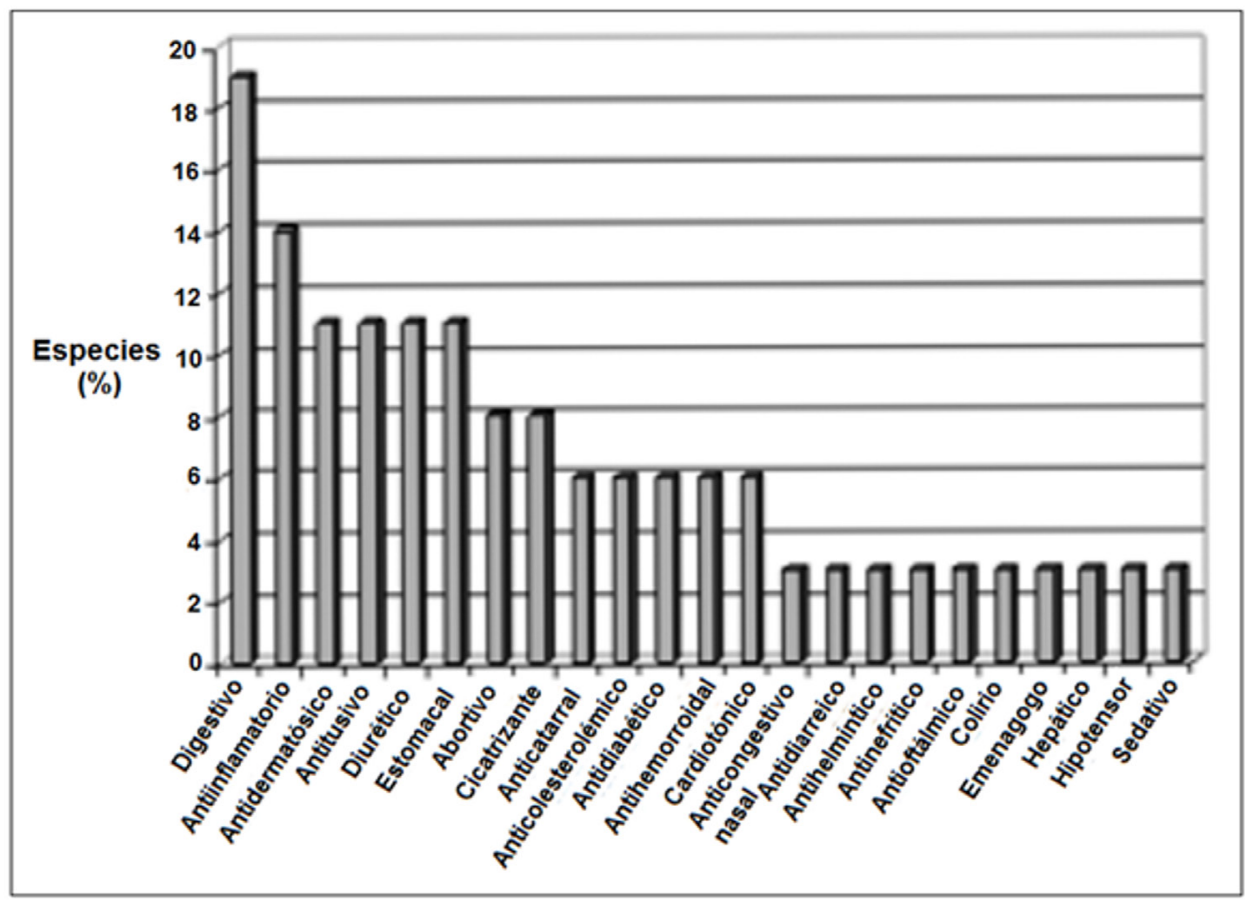

Fig. 4. Porcentaje de especies según sus usos. 
Tabla 2. Número y porcentaje de especies usadas para trastornos de los diferentes sistemas o aparatos.

\begin{tabular}{|lcc|}
\hline Sistema o Aparato & $\begin{array}{c}\text { Especies } \\
\mathbf{N}^{\mathbf{0}}\end{array}$ & $\begin{array}{c}\text { Porcentaje } \\
\mathbf{\%}\end{array}$ \\
\hline Digestivo & 18 & 50 \\
Epidérmico & 7 & 19 \\
Respiratorio & 7 & 19 \\
Circulatorio & 5 & 14 \\
Muscular & 5 & 14 \\
Urinario & 5 & 14 \\
Reproductivo & 4 & 11 \\
Ocular & 2 & 6 \\
Nervioso & 1 & 3 \\
\hline
\end{tabular}

Tabla 4. Modo de preparación de las especies vegetales con uso medicinal local y porcentaje.

\begin{tabular}{|lcc|}
\hline \multicolumn{1}{|c}{ Modo de Preparación } & $\begin{array}{c}\text { Especies } \\
\mathbf{N}^{\mathbf{0}}\end{array}$ & $\begin{array}{c}\text { Porcentaje } \\
\mathbf{\%}\end{array}$ \\
\hline Infusión & 19 & 53 \\
Decocción & 8 & 22 \\
Hoja picada & 2 & 5 \\
Macerado acuoso & 2 & 5 \\
Jarabe (azúcar blanca) & $\mathbf{1}$ & 3 \\
Jarabe (azúcar negra y miel) & 1 & 3 \\
Pulpa & 1 & 3 \\
Rodajas de tubérculo & 1 & 3 \\
Tintura & 1 & 3 \\
\hline
\end{tabular}

y Paulino, y localidades de Palo Blanco y Los Talas (Hernández et al., 2009, 2010, 2013). Se concluye que los habitantes del área de estudio utilizan con frecuencia el recurso vegetal medicinal de hábito herbáceo, empleando las hojas para la elaboración de infusiones calientes administradas por vía oral.

\section{Agradecimientos}

Se expresa el reconocimiento a los pobladores del área por permitir y acompañar las tareas realizadas durante las entrevistas.
Tabla 3. Partes de las especies vegetales con uso medicinal local y porcentaje.

\begin{tabular}{|lcc|}
\hline \multicolumn{1}{|c}{ Partes } & $\begin{array}{c}\text { Especies } \\
\mathbf{N}^{\mathbf{0}}\end{array}$ & $\begin{array}{c}\text { Porcentaje } \\
\mathbf{\%}\end{array}$ \\
Hoja & 27 & 75 \\
Parte aérea & 3 & 8 \\
Fruto & 2 & 5 \\
Planta entera & 1 & 3 \\
Flor & 1 & 3 \\
Tallo & 1 & 3 \\
Tubérculo & 1 & 3 \\
\hline
\end{tabular}

Tabla 5. Modos de administración de las especies vegetales con uso medicinal local y porcentaje.

\begin{tabular}{|lcc|}
\hline \multicolumn{1}{|c}{$\begin{array}{c}\text { Modo de } \\
\text { Administración }\end{array}$} & $\begin{array}{c}\text { Especies } \\
\mathbf{N}^{\mathbf{0}}\end{array}$ & $\begin{array}{c}\text { Porcentaje } \\
\mathbf{\%}\end{array}$ \\
Oral-caliente & 22 & 62 \\
Tópico-frío & 7 & 20 \\
Tópico-tibio & 2 & 6 \\
Inhalación-vapor & 1 & 3 \\
Oral-frío & 1 & 3 \\
Oral-masticación & 1 & 3 \\
Oral-tibio & 1 & 3 \\
\hline
\end{tabular}

Agradecemos a Marta Colares por el aporte bibliográfico y a los evaluadores anónimos las sugerencias brindadas que permitieron mejorar el mismo.

\section{Bibliografía}

AGARWAL, R., A. K. PANT \& O. PRAKASH. 2012. Chemical composition and biological activities of essential oils of Cinnamomum tamala, Cinnamomum zeylenicum and Cinnamomum camphora growing in Uttarakhand. InL. K. Khemani, M. M. Srivastava, Shalini Srivastava (eds.), Chemistry of Phytopotentials: Health, energy and environmental perspectives 18: 87-92, Springer.

ALIAGA MAMANI, P. A. 2013. Evaluación de la actividad antibacteriana "in vitro" del aceite esencial de hojas de Aloysia triphylla P. "Cedrón" frente a Escherichia coli ATTC 25922 y Staphylococcus aureus 25923. Tesis, Universidad Nacional Jorge 
Basadre Grohmann, Facultad de Ciencias, Escuela Académico Profesional de Biología-Microbiología TACNA-PERU. 134 pp.

ALONSO, J. R. \& C. DESMARCHELIER. 2005. Plantas medicinales autóctonas de la Argentina. Bases Científicas para su Aplicación en Atención Primaria de la salud. L.O.L.A., Buenos Aires. 663 pp.

ALONSO PAZ, E., M. J. BASSEGODA \& F. FERREIRA. 2007. Yuyos: uso racional de las plantas medicinales. Fin de Siglo, Montevideo. 224 pp.

ARANGO MEJÍA, M. C. 2006. Plantas medicinales: botánica de interés médico. Medicina indígena colombiana, según los cronistas y viajeros. Ed.: M. C. Arango Mejía. Colombia. 437 pp.

ARTECHE GARCÍA, A. 1992. Fitoterapia. Vademecum de prescriptions. Bilbao. CITA. 835 pp.

ARTURI M. F., C. A. PÉREZ, M. HORLENT, J. F. GOYA \& S. TORRES ROBLES. 2006. Las especies vegetales invasoras en los talares bonaerenses. En: Talares bonaerenses y conservación. E. Mérida \& J. Athor (eds.). Fundación de Historia Natural Félix de Azara. Universidad Maimónides, Buenos Aires, pág. 37.

BÁEZ MORALES, R., N. BRUCE DIAGO, S. PROVEYER DERICH \& M. C. HERNÁNDEZ. 1995. Evaluación antibacteriana de extractos de cítricos de la provincia de Ciego de Avila, consulta 03/03/2014, http://bvs.sld.cu/revistas/mciego/ vol1_01_95/a4_v1_0195.html

BARBOZ̄A, G. E., J. J. CANTERO, C. NÚÑEZ, A. PACCIARONI \& L. ARIZA ESPINAR. 2009. Medicinal Plants: A general review and a phytochemical and ethnopharmacological screening of the native Argentine Flora. Kurtziana 34: 7-365.

BARRERA C. A. \& G. E. ACOSTA. 2013. Actividad antibacteriana y determinación de la composición química de los aceites esenciales de romero (Rosmarinus officinalis), tomillo (Thymus vulgaris) y cúrcuma (Curcuma longa) de Colombia. Rev. Cubana Plant. Med. 18: 237-246.

BOERIS, M. A., R. E. TOSO, G. J. OCHOA, D. A. MANSO, M. E. CUCCOLO \& M. I. SKLIAR. 2002. Estudio de las propiedades antiinflamatorias de Marrubium vulgare. Ciencia Veterinaria. Facultad de Ciencias Veterinarias. U.N.L. Pam. 4: 1-6.

BUET, F., E. A. ULIBARRI \& J. A. HURRELL. 2010. Las huertas familiares en la Isla Paulino (Buenos Aires, Argentina). EnM. L., Pochettino, A. H. Ladio \& P. M. Arenas (eds.), Tradiciones y Transformaciones en Etnobotánica, La Plata, Argentina. Ed. CYTED. San Salvador de Jujuy. 561 pp.

BUET, F., F. PUENTES, E. A. ULIBARRI \& J. A. HURRELL. 2011. Estudio etnobotánico de los huertos familiares de la Isla Santiago (Buenos Aires, Argentina). XXXIII Jornadas Argentinas de Botánica, Bol. Soc. Arg. Bot. 46 (Supl.): 130.

CABRERA, A. L. 1949. Las comunidades vegetales de los alrededores de La Plata Provincia de Buenos Aires, República Argentina. Lilloa 20: 269-274.

CABRERA, A. L. \& G. DAWSON. 1944. La selva marginal de Punta Lara en la ribera argentina del Río de La Plata. Revista Museo de La Plata, Secc. Bot. 5: 267-382.

CABRERA, A. L. \& E. M. ZARDINI. 1978. Manual de la Flora de los alrededores de Buenos Aires. Ed. Acme, Buenos Aires. 758 pp.

CASTAÑO, P. H. I., C. G. GELMY, J. E. ZAPATA M. \& S. L. JIMÉNEZ R. 2010. Actividad bactericida del extracto etanólico y del aceite esencial de hojas de Rosmarinus officinalis L. sobre algunas bacterias de interés alimentario. Vitae 17: 149-154.

CATÁLOGO DE LAS PLANTAS VASCULARES DE FLORA DE LA REPÚBLICA ARGENTINA. 2015. http://www.floraargentina.edu.ar/[acceso: abril 2015]

CERDEIRAS, M. P., F. HORVATH, A. MONTFALCON \& A. VÁZQUEZ. 2007. Estudio de los metabolitos antimicrobianos de Xanthium cavanillesii. Bol. Latinoam. Caribe Plant. Med. Aromát. 6: 334.

CONSOLINI, A. E., A. BERNARDI, M. A. ROSELLA \& M. G. VOLONTÉ. 2011. Antispasmodic effects of Aloysia polystachya and A. gratissima tinctures and extracts are due to non-competitive inhibition of intestinal contractility induced by acethylcholine and calcium. Rev. Bras. Farmacogn. 21: 889-900.

CHERUSE, J. J., O. A. N. BALDINI \& L. B. BLANCH. 1983. Ensayo farmacodinámico preliminar de Euphorbia serpens H.B.K. (Euphorbiaceae). Acta Farm. Bonaerense 2: 19-22.

DE LA PAZ NARANJO M., M. A. MACEIRA CUBILES, A. SALVADÓ CORRAL \& C. GONZÁLEZ CAMPOE. 2006. Actividad antiparasitaria de una decocción de Mentha piperita L. Rev. Cubana Med. Milit. 35: 1-4.

DE MARTINO, L., V. DE FEO, C. FORMISANO, E. MIGNOLA \& F. SENATORE. 2009. Chemical composition and antimicrobial activity of the essential oils from three chemotypes of Origanum vulgare L. ssp. hirtum (Link) Ietswaart growing wild in Campania (southern Italy). Molecules 14: 2735 2746.

DE PAULA, F., M. LOCKS, M. BELTRÃO \& M. AMARAL. 2002. Estudio preliminar etnobotânico de plantas de uso medicinal na Região arqueológica de Central-Bahia, Brasil. Tercer Congreso Virtual de Antropología y Arqueología. Equiponaya.com.ar

DELUCCHI, G. \& S. TORRES ROBLES. 2006. Las especies vegetales invasoras en los talares bonaerenses. En E. Mérida \& J. Athor (eds.), Talares bonaerenses y conservación. Fundación de Historia Natural Félix de Azara. Universidad Maimónides, Buenos Aires, pág. 146.

DIMITRI, M. J. (ed.). 1978. Enciclopedia Argentina de agricultura y jardinería, 3ra. ed., Acme, Buenos Aires. Tomo $1 \mathrm{~V}$ 1: 653 pp. 
DIMITRI, M. J. (ed.). 1980. Enciclopedia Argentina de agricultura y jardinería, 3ra. ed., Acme, Buenos Aires. Tomo 1 V 2: 657-1161.

DOMÍNGUEZ FERNÁNDEZ, R. N., I. ARZATE VÁZQUEZ, J. J. CHANOMA PÉREZ, J. S. WELTI CHANES, J. S. ALVARADO GONZÁLEZ, G. CALDERÓN DOMÍNGUEZ, V. GARIBAY FEBLES \& G. F. GUTIÉRREZ LÓPEZ. 2012. El gel de Aloe vera: estructura, composición química, procesamiento, actividad biológica e importancia en la industria farmacéutica y alimentaria. Rev. Mex. Ing. Quím. 11: 23-43.

ESQUIVEL FERRIÑO, P., G. PEDROZA-CANTÚ, N. SANDOVAL-MONTENEGRO, R. E. MATAMARTÍNEZ, L. MENDOZA-OBREGÓN \& I. BALDERAS-RENTERÍA. 2010. Ensayo químico dirigido y estudio del efecto antimicrobiano in vitro de algunos condimentos empleados en la cocina mexicana. RESPYN 10: 23-25.

GARCÍA GÓMEZ, L. J. \& F. J. SÁNCHEZ-MUÑIZ. 2000. Revisión: Efectos cardiovasculares del ajo (Allium sativum). Arch. Latinoamer. Nutr. 50: 25-46.

GARCÍA VARGAS, K. 2010. Usos terapéuticos de la herbolaria mexicana. "El temazcal curativo y el uso tradicional de las plantas". Tesina Universidad Autónoma de Chapingo, Programa Universitario de Medicina Tradicional y Terapéutica Naturista. 47 pp.

GUPTA, M. 1995. 270 Plantas medicinales Iberoamericanas. CYTED-SECAB, Santafé de Bogotá, Colombia. 617 pp.

GUZELDAG, G., L. KADIOGLU, A. MERCIMEK \& F. MATYAR. 2014. Preliminary examination of herbal extracts on the inhibition of Helicobacter pylori. Afr. J. Tradit. Complement Altern. Med. 11: 93-96.

HERNÁNDEZ, M. P. \& A. M. ARAMBARRI. 2011. Recursos fitoterapéuticos y comportamiento poblacional en dos sectores de la ribera rioplatense de Berisso, Buenos Aires, Argentina. Bonplandia 20: 137-148.

HERNÁNDEZ, M. P., S. M. COLARES \& S. M. CIVITELLA. 2009. Plantas utilizadas en medicina popular en un sector del Partido de Berisso, Buenos Aires, Argentina. Bol. Latinoam. Caribe Plant. Med. Aromát. 8: 435-444.

HERNÁNDEZ, M. P., S. M. CIVITELLA \& V. G. ROSATO. 2010. Uso medicinal popular de plantas y líquenes de la Isla Paulino, Provincia de Buenos Aires, Argentina. Bol. Latinoam. Caribe Plant. Med. Aromát. 9: 258-268.

HERNÁNDEZ, M. P., M. C. NOVOA, S. M. CIVITELLA, D. MASON \& A. OVIEDO. 2013. Plantas usadas en medicina popular en la Isla Santiago, Buenos Aires, Argentina. Bol. Latinoam. Caribe Plant. Med. Aromát. 12: 385-399.

HURRELL, J. A., E. A. ULIBARRI, F. C. BUET \& M. L. POCHETTINO. 2011. Huertos familiares periurbanos de las costas de Ensenada, Berisso y de la Isla Martín García (Buenos Aires, Argentina). Bonplandia 20: 213-229.

JIMOH, F., A. ADEDAPO, A. ALIERO \& A. AFOLAYAN. 2010. Polyphenolic and biological activities of leaves extracts of Argemone subfusiformis (Papaveraceae) and Urtica urens (Urticaceae). Int. J. Trop. Biol. 58: 1517-1531.

KOBAYASHI, Y., R. TAKAHASHI \& F. OGINO. 2005. Antipruritic effect of the single oral administration of german chamomile flower extract and its combined effect with antiallergic agents in ddY mice. J. Etnopharmacol. 101: 308-312.

LAHITTE, H. B., HURRELL, J. A., K. MEHLTRETER, M. J. BELGRANO, L. S. JANKOWSKI, M. P. HALOUA \& G. CANDA. 1997. Plantas de la costa. L.O.L.A., Buenos Aires. 200 pp.

LAHitTE, H. B., HURRELL, J. A., M. J. BELGRANO, L. S. JANKOWSKI, P. HALOUA \& K. MEHLTRETER. 1998. Plantas medicinales rioplatenses. L.O.L.A., Buenos Aires. 240 pp.

LAMBERTI, R. O., H. O. TROIANI, P. E. STEIBEL, R. E. TOSO, M. A. BOERIS, L. M. GINO, C. D. CALVO, G. BERTORELLO MASCARÓ, M. GIRAUDO \& G. GENERO. 2009. Cribado antihelmíntico de plantas recolectadas en la provincia de La Pampa. Ciencia Veterinaria 11: 26-31.

LANGMEAD, L., R. MAKINS \& D. RAMPTON. 2003. Anti-inflammatory effects of Aloe vera gel in human colorectal mucosa in vitro. Aliment. Pharmacol. Ther. 19: 521-527.

MEYRE SILVA, C., R. A. YUNES, V. SCHLEMPER, F. CAMPOS BUZZI \& V. CECHINEL-FILHO. 2005. Analgesic potential of marrubiin derivatives, a bioactive diterpene present in Marrubium vulgare (Lamiaceae). Farmaco 60: 321-326.

NAYAK, B., S. RAJU \& A. RAO. 2007. Wound healing activity of Matricaria recutita $\mathrm{L}$. extract. J. Wound Care 3: 298-302.

OLIVER-BEVER, B. 1986. Medicinal Plants in Tropical West Africa. London: Cambridge University Press. 168 pp.

PEREZ, C., J. R. CANAL \& M. D. TORRES. 2003. Experimental diabetes treated with Ficus carica extract: effect on oxidative stress parameters. Acta Diabetol. 40: 3-8.

PÉREZ GUTIÉRREZ, R., G. YESCAS LAGUNA \& A. WALSKOUSKI. 1985. Diuretic activity of Mexican Equisetum. J. Ethnopharmacol. 14: 269-272.

PINTO DÁVALOS J. \& Z. BUSTAMANTE GARCÍA. 2008. Evaluación de la actividad gastroprotectora de los extractos de llantén (Plantago major). BIOFARBO 16: 36-41.

POCHETTINO, M. L. 2015. Botánica económica. Las plantas interpretadas según tiempo, espacio y cultura. Soc. Argent. Bot. , Buenos Aires, 448 pp.

POCHETTINO, M. L., M. R. MARTÍNEZ, B. ITTEN \& M. ZUCARO. 1997. Las plantas medicinales como 
recurso terapéutico en una población urbana: estudio etnobotánico en Hernández (Pdo. La Plata, Prov. Buenos Aires, Argentina). Parodiana 10: 141-152.

POCHETTINO, M. L., P. ARENAS, D. SÁNCHEZ \& R. CORREA. 2008. Conocimiento botánico tradicional, circulación comercial y consumo de plantas medicinales en un área urbana de Argentina. Bol. Latinoam. Caribe Plant. Med. Aromáticas 7: 141-148.

RHAMAN, S. M. A., S. A. ABD-ELLATIF, S. F. DERAZ \& A. A. KHALIL. 2011. Antibacterial activity of some wild medicinal plants collected from western Mediterranean coast, Egypt: natural alternatives for infectious disease treatment. Afr. J. Biotechnol. 10: 10733-10743.

RAMAKANT, S., S. MANVENDRA, S. B. KASTURE, A. POOJA \& K. C. SAMANTA. 2012. Evaluation of antiulcer activity of leaf extracts of Laurus nobilis L. J. Chronother. Drug Deliv. 3: 99-107.

REMES TROCHE, J. M., O. GÓMEZ-ESCUDERO, J. R. NOGUEIRA-DE ROJAS, R. CARMONASÁNCHEZ, J. PÉREZ-MANAUTA, A. LÓPEZCOLOMBO, J. L. SANJURJO-GARCÍA, A. NOBLE-LUGO, J. A. CHÁVEZ-BARRERA \& M. GONZÁLEZ-MARTÍNEZ. 2010. Tratamiento farmacológico del síndrome de intestino irritable: revisión técnica. Rev. Gastroenterol. Mex. 75: 44-66.

REYES MUNGUÍA, A., D. ZAVALA-CUEVAS, \& A. ALONSO-MARTÍNEZ. 2012. Perejil (Petroselinum crispum): compuestos químicos y aplicaciones. TLATEMOANI. Rev. Acad. Investigación 11: 2-18.
RONDINA, R. V. D., A. L. BANDONI \& J. D. COUSIO. 2008. Especies medicinales argentinas con potencial actividad analgésica. Dominguezia 24: 47-69.

SALAZAR ARANDA, R., Y. C. DE LA TORRERODRÍGUEZ, B. A. ALANÍS-GARZA, L. A. PÉREZ-LÓPEZ \& N. WAKSMAN. 2009. Evaluación de la actividad biológica de productos herbolarios comerciales. Rev. Medicina Universitaria 11: 156164.

SANDOVAL-VEGAS, M., O. HUAMÁN-GUTIÉRREZ, R. ORÉ-SIFUENTES, A. LOLI-PONCE, S. AYALAPÍO. 2010. Efecto antioxidante y citoprotector del Solanum tuberosum (papa) en la mucosa gástrica de animales de experimentación. An. Fac. med. 71: 147-52.

SHARMA, D., V. K. BHATIA, S. PATIL \& P. C. SHARMA 2013. Antimicrobial activity of selected cryptogams from Solan región. International J. Biol. Pharm. Res. 4: 448-454.

SINGH, C. H., S. SINGH, C. PANDE, G. TEWARI, V. PANDE \& P. SHARMA. 2013. Exploration of antimicrobial potential of essential oils of Cinnamomum glanduliferum, Feronia elephantum, Bupleurum hamiltonii and Cyclospermum leptophyllum against foodborne pathogens. Pharm. Biol. 51: 1607-1610.

TROPICOS. ORG. MISSOURI BOTANICAL GARDEN. 2015. http://www.tropicos.org/ [acceso: abril 2015].

VÁZQUEZ, B., G. AVILA \& D. SEGURA. 1996. Antiinflammatory activity of extracts from Aloe vera gel. J. Ethnopharmacol. 55: 69-75.

Original recibido el 22 de abril de 2015; aceptado el 15 de octubre de 2015. 